Research Article

\title{
Molecular Dynamic Simulation and Docking of Cyclophilin A Mutants with its Potential Inhibitors
}

\author{
Alireza Mohebbi ${ }^{1}{ }^{2},{ }^{3}$, Azam Mirarab ${ }^{3}$, Reyhane Shaddel ${ }^{2}$, Mahnaz Shafaei Fallah ${ }^{3}$, Ali \\ Memarian *4 \\ 1 Stem Cell Research Center, School of Medicine, Golestan University of Medical Sciences, \\ Gorgan, Iran \\ 2 Student Research Committee, School of Medicine, Golestan University of Medical Sciences, \\ Gorgan, Iran \\ 3 Department of Microbiology, School of Medicine, Golestan University of Medical Sciences, \\ Gorgan, Iran \\ 4 Golestan Research Center of Gastroenterology and Hepatology, Golestan University of \\ Medical Sciences, Gorgan, Iran \\ *Correspondence: Ali Memarian, Golestan Research Center of Gastroenterology and \\ Hepatology, Golestan University of Medical Sciences, Gorgan, Iran \\ Email: alimemarian@goums.ac.ir
}

Tel: +981732421717

Received April 1, 2021

Accepted May 17, 2021

\begin{abstract}
Background and objectives: Cyclophilin A (CypA) is involved in various human biological processes. Its role in many pathological conditions makes it a promising target for treating human diseases, such as viral infections. The aim of the present study was to investigate docking of CypA mutants with its potential inhibitors using molecular dynamic simulation ((MDS).

Methods: The crystallographic structure of CypA was extracted from the protein database (PDB). Important CypA substitutions were obtained from the literature. CypA inhibitors were taken from chemical databases. The affinity and binding sites of the compounds to CypA and its mutants were also scaled through Autodock Vina. Root-mean-square deviation (RMSD), radios gyration, Lenard-jones potential, and hydrogen bonding were investigated by using MDS for 600 ps.

Results: The findings revealed that SangfA and HBF-0259 had more affinity to the CypA ($7.8 \mathrm{Kcal} / \mathrm{mol}$ and $-7.5 \mathrm{Kcal} / \mathrm{mol}$, respectively). Conformational changes were observed in CypA W121A/F mutants. SangfA complexed with CypA and its mutants had relatively stable RMSD. Higher Lenard-Jones potential has been observed in the interaction of SangfA to W121A, HBF0259 to M61, and SCY-635 to H70F. The SangfA had a higher HBs ratio with CypA.

Conclusion: Given the higher affinity of SangfA and HBF-0259 to CypA and its mutants, they would influence the stability of the protein. RMSD analysis revealed that SangfA is probably ligated to CypA and its mutants, which are relatively stable. Substitution at W121 residue would reduce inhibitor binding to CypA.
\end{abstract}

Keywords: HBF-0259; Cyclophilin A; Sanglifehrin A; CypA inhibitors; Molecular Dynamic Simulation

\section{DOI: 10.29252/Jcbr.5.2.26}

\section{(c) (i) (8)}

This work is licensed under a Creative Commons Attribution 4.0 License.

(C) The authors 


\section{INTRODUCTION}

Cyclophilin A (CypA) is a cytosolic protein with immunophilin and peptidyl-prolyl cis to trans isomerase (PPIase) activities, acting on the folding of proteins containing GP motif (1). The protein is comprised of two independent PPIase activity and drug binding domains (2). Some hydrophobic (R55) and hydrophilic residues (F60 and H126) are located in the CypA side chain, which is involved in the PPIase activity (2).

The role of CypA in several important viral infections has been reviewed ( $\underline{3})$. As an immunophilin, CypA is the target of a few immunosuppressive drugs. For example, cyclosporin A (CsA) analog, SCY-635 has shown significant anti-hepatitis $\mathrm{C}$ virus (HCV) activity via suppressing viral replication and inducing innate immunity response (4-ㅡㅇ). Therefore, the CypA ligand binding site is a potent target for drug discovery and treatment of human diseases $(\underline{7}, \underline{8})$. Antiviral effects of inhibitors make СурA a potent target for further specific drug discovery and development.

The ligand-binding site of CypA is a good target for drug discovery and investigation of the development of human viral infections. Immunosuppressive drugs, including CsA, SangfA, Alisporivir, NIH811, and SCY-635, can be used to fulfill this approach (9). Additionally, it has been predicted that HBF-0259 can bind to CypA and inhibit hepatitis $B$ virus (HBV) surface antigen (HBsAg) secretion (9). However, the mechanism of action of those potential inhibitors is not known.

Molecular dynamic simulation (MDS) and molecular docking are two valuable approaches for predicting the binding of small molecules and their relative affinity to their targets (10). MDS provides measurements for the evaluation of receptor conformational and flexibility changes due to the binding of ligands. It also provides further tools for assessing the affinity of ligands to their receptors. Furthermore, molecular dynamics simulations have been used to evaluate the allosteric binding sites between the activator and CypA $(\underline{11}, \underline{12})$.
Our study aims to investigate the effect of substitutions at CypA hydrophobic PPIase or ligand binding site domains on changes of CypA stability and its interaction energy with potential inhibitors in 600ps.

\section{MATERIALS AND METHODS \\ Receptor/inhibitor preparations}

The crystallographic structure of CypA (PDB ID: 1BCK) was provided from the Protein Data Bank (http://www.rcsb.org/pdb) (13, 14). The structure was cleaned from non-specific residues and water molecules by using UCSF Chimera 1.10.2 (15). Chemical structure of known CypA inhibitors and ligands, cyclosporin (Csp), alisporivir, NIM811, SCY-635, and sanglifehrin A (Sangf-A), were obtained from the PubChem database (16). The chemical structure of HBF-0259 was computationally drawn as described previously (). All structural energies were minimized using the Swiss-pdb Viewer (17).

\section{Prediction of toxicity of CypA inhibitor}

Toxicity Estimation Software Tool (T.E.S.T) has been used for predicting toxicity by applying 2D descriptors of the inhibitors (U.S. Environmental Protection Agency.) Accordingly, the pre-existed consensus method provided by the software was applied for analysis of Fathead minnow (Fm) LC50 (96hr), Daphnia magna (Dm) LC50 (48hr), Tetrahymena pyriformis (Tp) IGC50 (48hr), oral rat LD50, bioaccumulation factor (BioF), and developmental toxicity

\section{In silico site-specific mutagenesis and molecular docking}

The Dunbrak rotamer library was used to substitute targeted amino acid residues with hydrophobic Phenylalanine. This change will have fewer structural movements before molecular docking and dynamic simulation $(\underline{18}, \underline{19})$. The most probable amino acid conformation provided by the UCSF Chimera software was used to substituting 
desired amino acids. After mutagenesis, all atomic clashes were resolved using UCSF Chimera's energy minimization with its default settings. In addition to the previously described amino acids (9), two additional substitutions W121F and W121A were constructed, which have been reported to confer 75-200 fold CsA affinity reductions (20).

The Autodock Vina software was employed for the molecular docking study (21). The grid-box was coordinated to encompass the receptor (CypA) using the Autodock Tools 1.5.6 (Molecular Graphics Laboratory, the Scripps Research Institute). Furthermore, the ligands were prepared for docking by using the Autodock Tool.

\section{Molecular dynamic simulation}

The simulation of CypA in the presence and absence of ligand was performed using the GROMACS 5.1.4 software (22). The topology of the CypA was created using GROMACS utilities, whereas the topology of the ligand was generated using the PRODRG server in the framework of the GROMOS96 43a1 force field $(\underline{23}, \underline{24})$. All starting structures were solvated in a simple point charge water box under periodic boundary conditions using a $1.0 \mathrm{~nm}$ distance from the protein to the box faces. The system was then neutralized by $\mathrm{Cl}-$ or $\mathrm{Na}+$ ions. Following steepest descent energy minimization, the systems were equilibrated under NVT (constant number of particles, volume, and temperature) for $60 \mathrm{ps}$ at $300^{\circ} \mathrm{K}$, followed by $60 \mathrm{ps}$ under NPT (constant number of particles, pressure, and temperature) conditions. The electrostatic interactions were treated using the Particle Mesh Ewald (PME) method. Finally, 0.6ns MD was performed to analyze the stability of each system. MD simulation was done on a Core i7 system containing NVidia GeForce Graphical Processing Unit (GPU).

\section{Statistical analysis}

Variations of interaction energies and total affinity of inhibitors to CypA wild type and mutants were analyzed using the t-test. All statistical analyses were performed by using $\mathrm{R}$ statistics 3.3.1 packages at significance of 0.05 (25). One-way ANOVA test was performed to evaluate variance variations of RMSD and radius of gyration $(\mathrm{Rg})$ for CypA and its mutant in either unbounded or complex with the ligands.

\section{RESULTS}

\section{Toxicity estimations}

2D molecular structure of ligands was introduced to the TEST software for toxicity prediction. As shown in table 1, peptidebased CypA inhibitors, Alisporivir, CsA, NIM-811, SCY-635, and SangfA have almost the same toxicity, and HBF-0259 has more toxicity properties. However, the evaluated toxicities were not significant. 
Table 1. Toxicity prediction of CypA ligands

\begin{tabular}{|c|c|c|c|c|c|c|c|c|}
\hline Ligands & Formula & $\begin{array}{c}\text { Fm } \\
(\mathrm{LC50})\end{array}$ & $\begin{array}{c}\text { Dm } \\
\text { (LC50) }\end{array}$ & $\begin{array}{c}\text { Tp } \\
\text { (IGC50) }\end{array}$ & $\begin{array}{l}\text { Oral rat } \\
\text { (LD50) }\end{array}$ & BioF & DevT & Mutagenicity $^{\mathrm{c}}$ \\
\hline Alisporivir $^{\mathrm{a}, \mathrm{b}}$ & C63H113N11O12 & 6.93 & 4.74 & 4.98 & 3.43 & 0.48 & 1 & 0 \\
\hline CsA & C62H111N11O12 & 6.93 & 4.22 & 4.98 & 3.1 & 0.48 & 1 & 0 \\
\hline HBF-0259 & $\mathrm{C} 17 \mathrm{H} 13 \mathrm{~N} 4 \mathrm{Cl} 2 \mathrm{~F}$ & 8.69 & 6.15 & 5.29 & 2.39 & 0.52 & 0.92 & 0.67 \\
\hline NIM-811 & C62H111N11O12 & 6.93 & 4.22 & 4.98 & 3.1 & 0.48 & 1 & 0 \\
\hline SCY-635 & C66H120N12O13S & 6.93 & 4.28 & $\mathrm{~N} / \mathrm{A}^{\mathrm{d}}$ & 3.43 & N/A & 0.67 & 0 \\
\hline SangfA & C60H91N5O13 & 6.93 & 4.31 & N/A & 2.38 & 0.38 & 0.67 & 0 \\
\hline & & & $\begin{array}{l}\log 10 \\
\text { the N } \\
\text { nes my } \\
\text { Not ap }\end{array}$ & $\begin{array}{l}\text { L) } \\
\text { t neight } \\
\text { hesis } \\
\text { ple }\end{array}$ & thod. & & & \\
\hline
\end{tabular}

CypA mutant RMSD and radios gyration ( $\mathrm{Rg}$ ) variations

Here, it was answered if the substitution of residues found in the binding site would cause any instability in CypA. For this purpose, RMSDs were compared to CypA as control. As shown in figure 1, the MD simulation of CypAs resulted in an increase in the RMSD over 10ns in most mutants. Among the variants, only W121A and W121F were relatively stable over time
$(\mathrm{P}>0.0)$. Other substitutions had clear significant increasing states. Exceptionally, the R55F mutant had significantly declining RMSD ( $<<0.0001)$.

The $\mathrm{Rg}$ in all structures was reduced in comparison with CypA (Figure 2). These variations were significant in substitutions G150F, K125F, and H70F. A decrease in Rg was continuous in two later substitutions over time. 


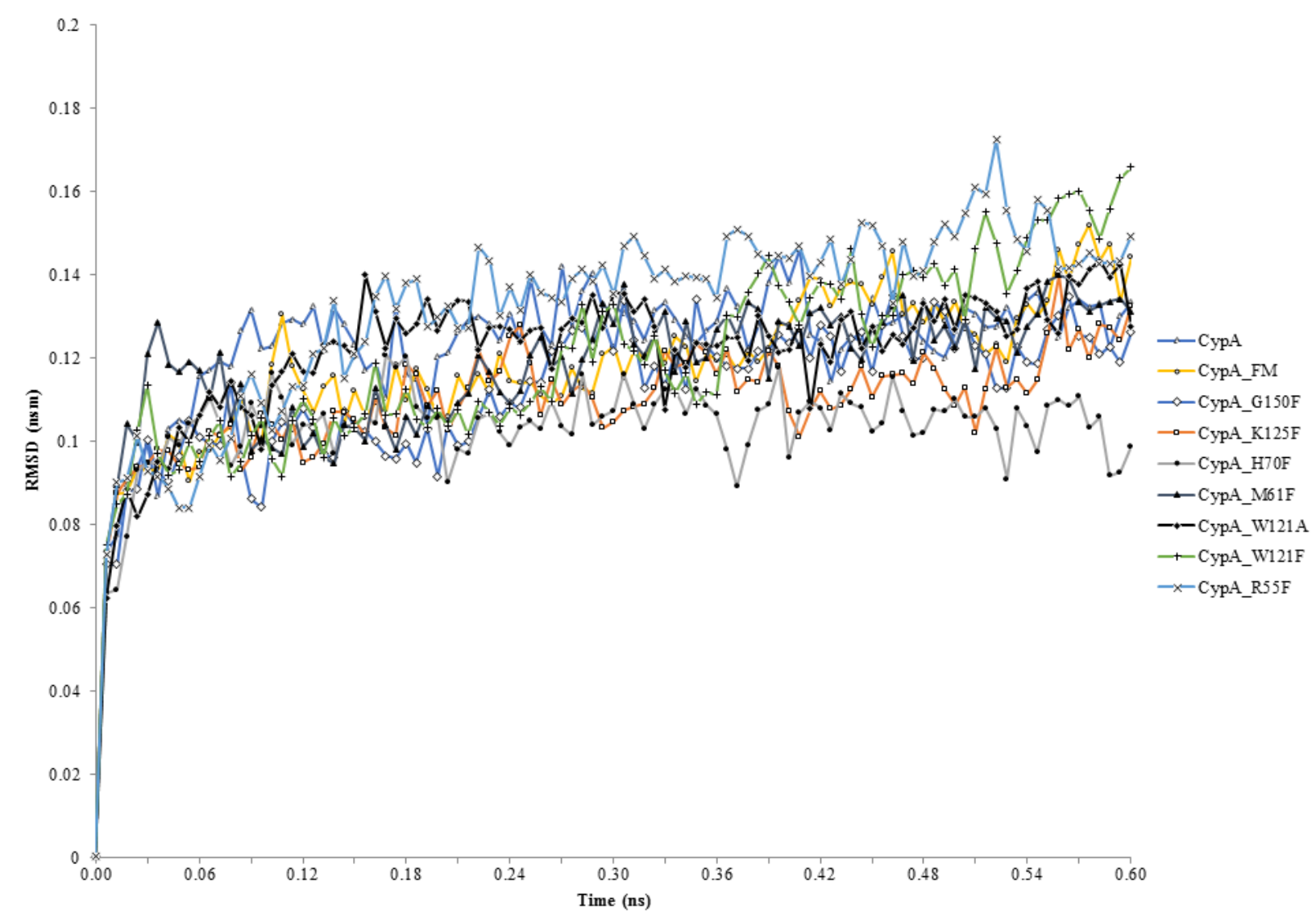

Figure 1. RMSD analysis of CypA variants. RMSD of CypA and its mutants has evaluated with GROMACS. CypA_R55F had the maximum RMSD mean (0.133 \pm 0.024$)$. Other RMSDs were $0.124 \pm 0.018,0.12 \pm 0.019,0.11 \pm 0.018,0.11 \pm 0.015,0.103 \pm 0.014,0.12 \pm 0.018,0.12 \pm 0.019$, and 0.12 \pm 0.024 for CypA, CypA_FM, CypA_G150F, CypA_K125F, CypA_H70F, CypA_M61F, CypA_W121A, and CypA_W121F, respectively. RMSD mean differences of CypA and its variants were significant $(F(8,900)=19.7, \mathrm{p}<0.0001)$. 


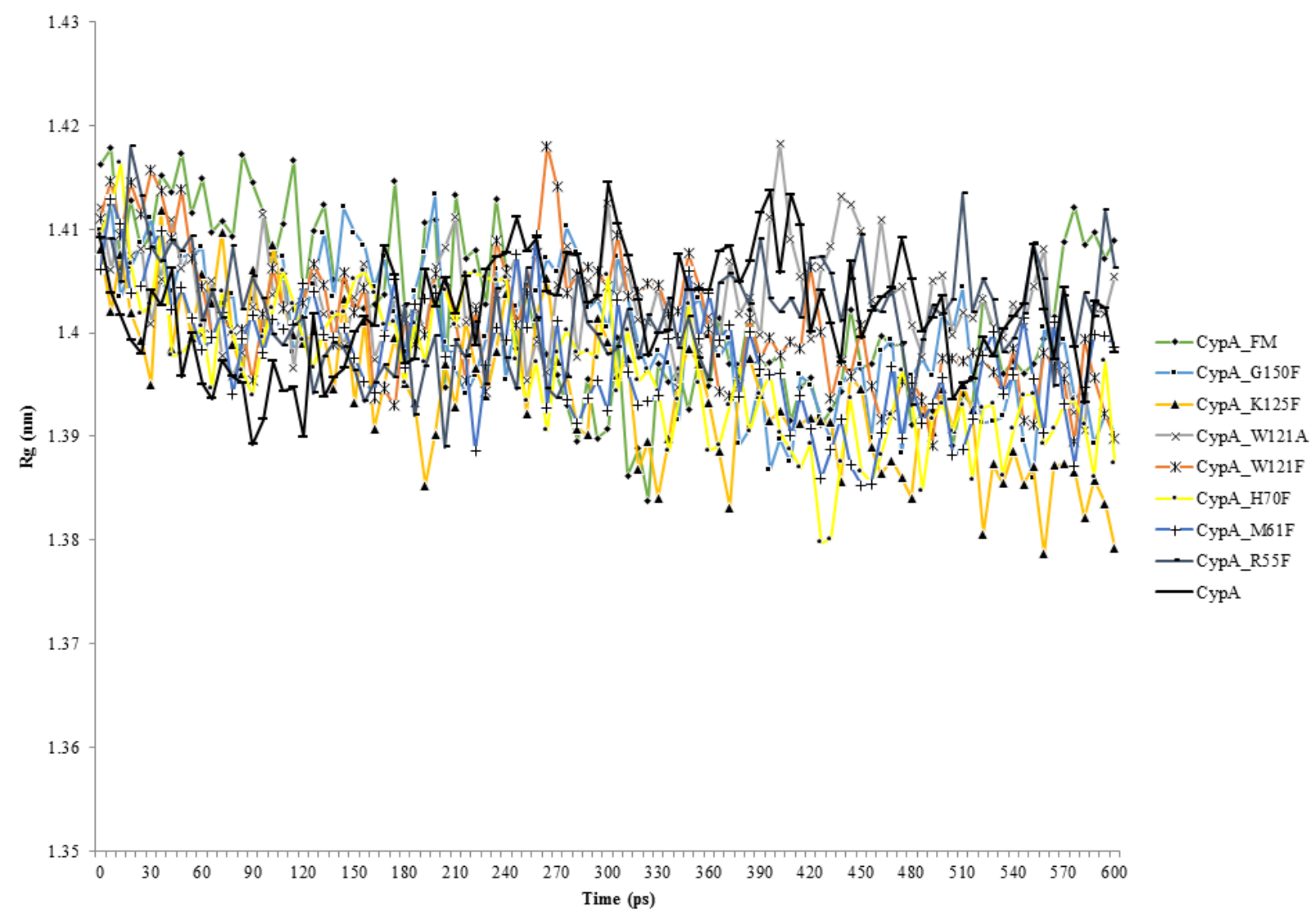

Figure 2. The Rg of CypA and its mutants. There are significant changes in the Rg value of CypA and its mutants $(\mathrm{F}(8,900)=22.96, \mathrm{p}<0.0001$

\section{Affinity of SangfA and HBF-0259 to CypA and its variants}

Binding affinities of the potent CypA inhibitors are shown in figure 3. It was observed that SangfA and HBF-0259 had maximum affinities to CypA $(-7.8 \mathrm{Kcal} / \mathrm{mol}$ and $-7.5 \mathrm{Kcal} / \mathrm{mol}$, respectively). SangfA had the highest interaction energy with CypA_R55F $(-9.7 \mathrm{Kcal} / \mathrm{mol} \pm 0.252)$ and the lowest interaction energy with CypA_H70F $(-7.8 \mathrm{Kcal} / \mathrm{mol})$. On the other hand, HBF0259 had higher binding energy to
CypA_M61F (-8.3Kcal/mol \pm 0.115$)$ compared with other mutants (Figure 3). The difference of SangfA binding energy to CypA R55F mutant was statistically significant $(\mathrm{p}<0.001)$. Furthermore, HBF0259 mean binding affinity to CypA wild type was significantly lower than that to G150F $(\mathrm{p}<0.01)$, M61F $(\mathrm{p}<0.001)$, and R55F ( $p<0.01)$. Other inhibitors' affinity and related analyses have been shown in supplemented materials (Supp1). 


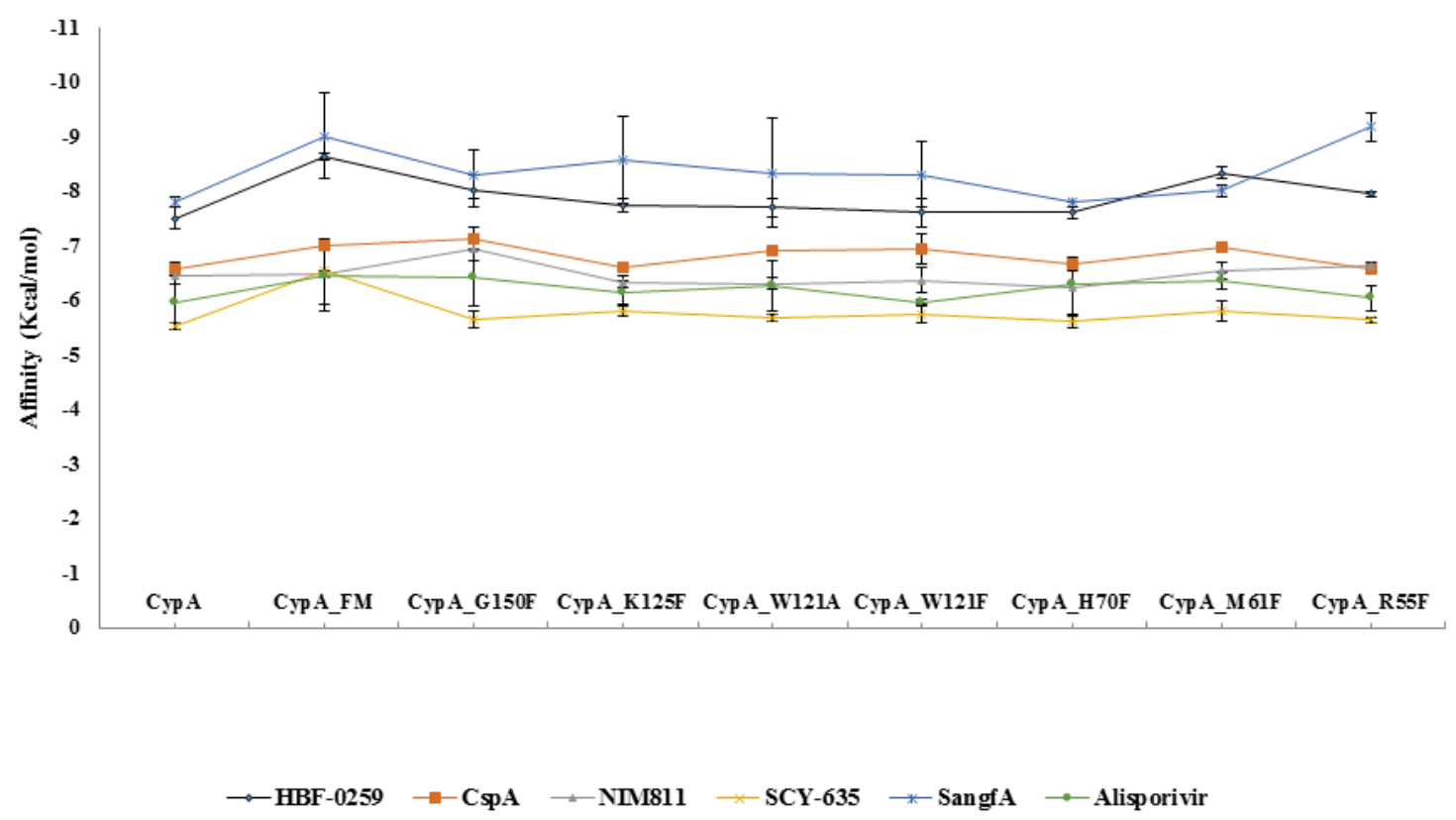

Figure 3. Ligands affinity to CypA and its variants. The t-test was performed on ligands' affinities to CypA. Docking was performed in triplicate for evaluating reproducibility.

Assessment of CypA stability in the presence of cognate inhibitors

Further analyses of CypA structural changes were evaluated using ligands with higher binding affinities (SangfA and HBF0259) compared to lower affinity (SCY635). As shown in figures $4 \mathrm{~A}$ and $4 \mathrm{~B}$, RMSD and $\mathrm{Rg}$ increased and decreased, respectively. RMSD changes over MD were significant $(\mathrm{F}(2,300)=25.265, \mathrm{p}<0.0001)$. However, $\mathrm{Rg}$ variance changes were not significant $(\mathrm{F}(2,300)=1.105, \mathrm{p}=0.33)$. Furthermore, MD simulation of CypA complexed with SangfA resulted in higher increased RMSD (0.1145 \pm 0.017$)$. In addition, $\mathrm{Rg}$ was more decreased in the CypA complexed with SCY-635 $(1.34 \pm 0.005)$. 


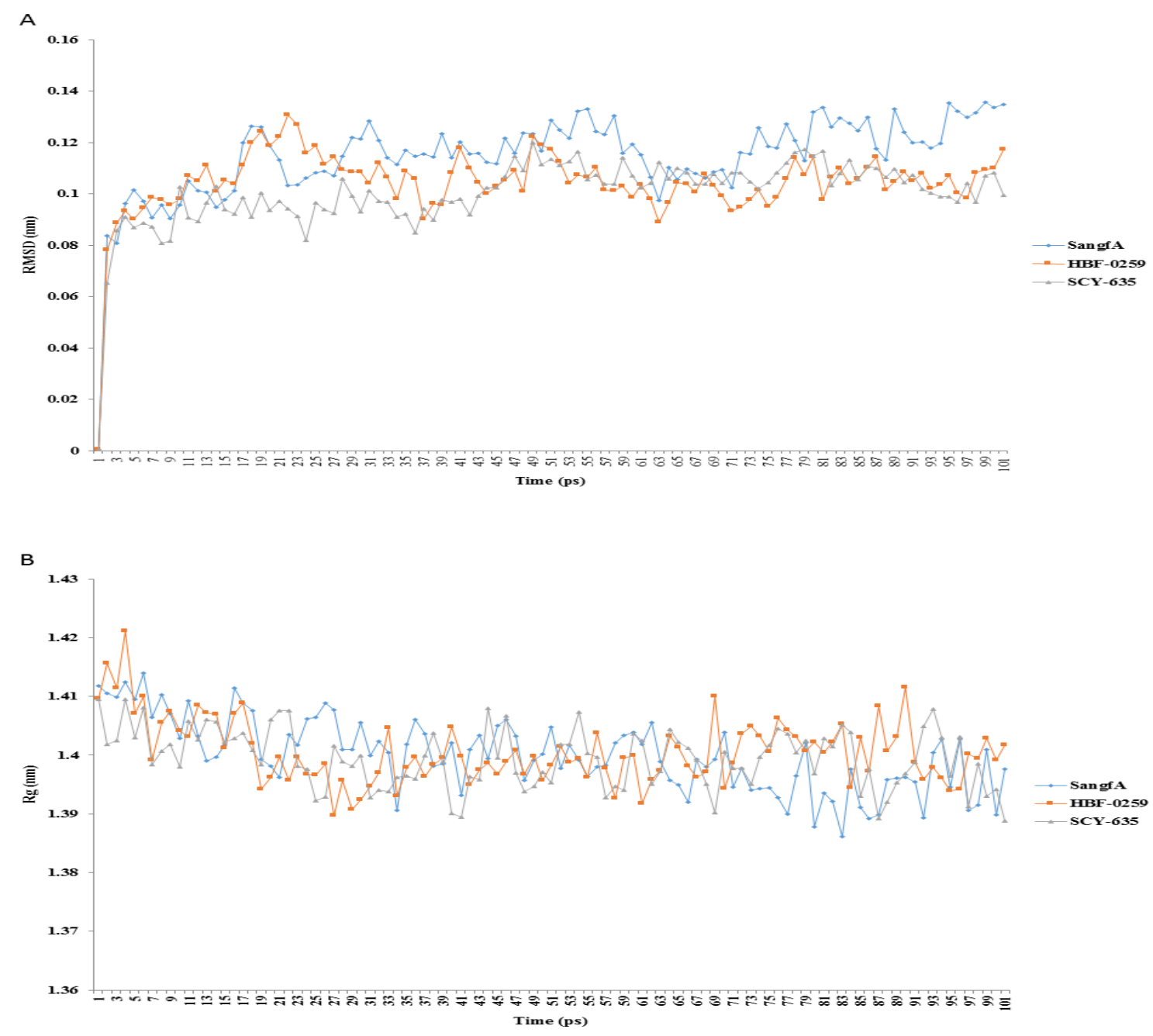

Figure 4. RMSD changes in CypA complexed with SangfA, HBF-0259, and SCY-635. B illustrates Rg variations of complexed CypA over 0.6ns.

MDS analysis of mutants in complex with SangfA, HBF-0259, and SCY-635

RMSD analysis revealed that SangfA complexed with CypA and its mutants have relatively stable RMSD. HBF-0259 and SCY-635 have almost the same RMSD changes excepted inW121A, H70F, and R55F (Figure 5A). Figure 5B shows that Rg of CypA in G150 and H70 mutants docked with SCY-635 was lower than that of the wild-type receptor. $\mathrm{Rg}$ of $\mathrm{HBF}-0259$ was higher than two other inhibitors and was at its peak in the G150 mutant. Furthermore, $\mathrm{Rg}$ amounts of CypA mutants complexed with SangfA had only minor changes (Figure 5B). Further RMSD data of ligands to other CypA mutants have been demonstrated separately in supplemented data (Supp2). 


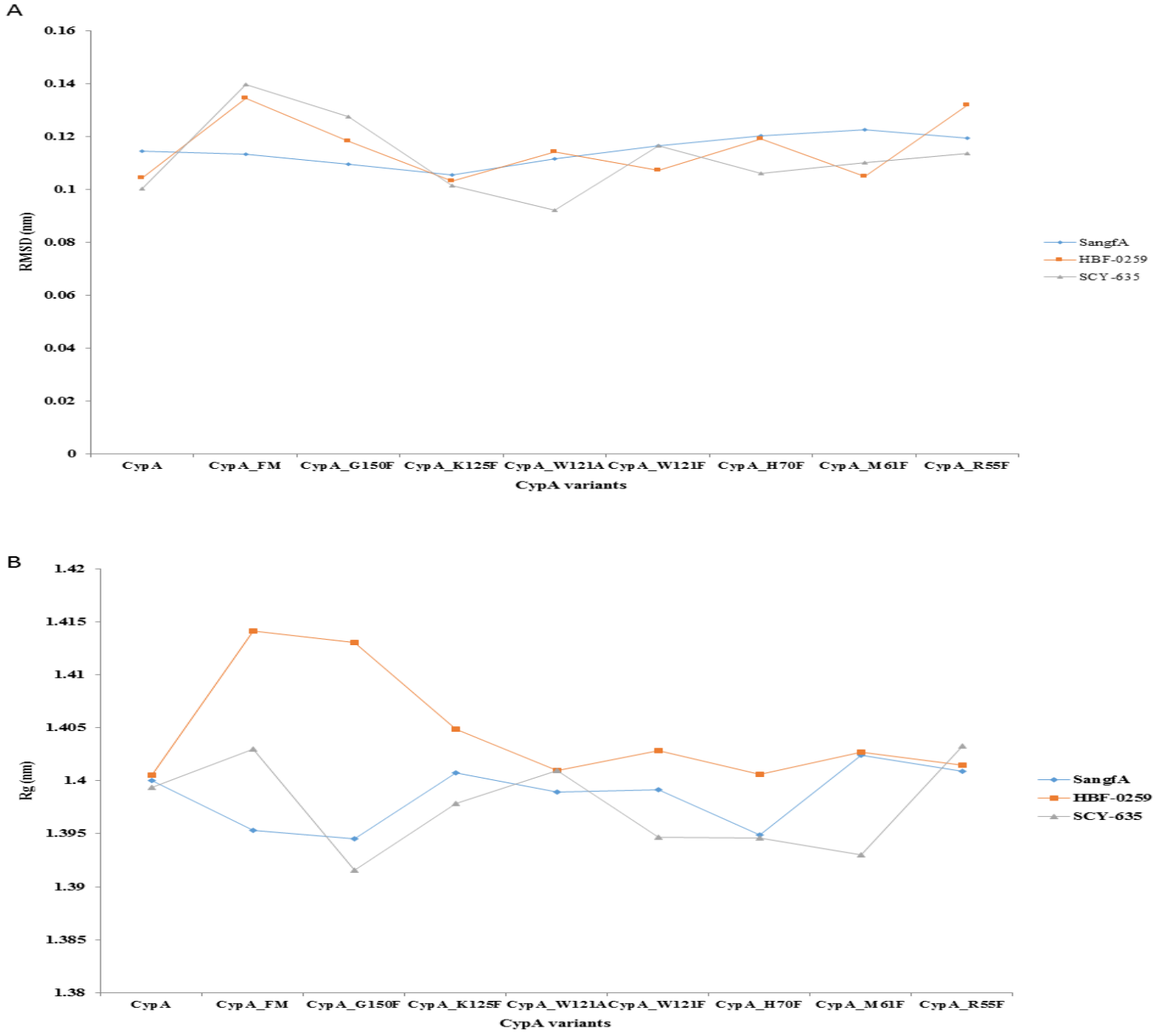

Figure 5. Mean RMSD (A) and Rg (B) changes in CypA mutants complexed with SangfA, HBF-0259, and SCY-635.

Higher Electrostatic and Leonard Jones Potential energies in the interaction of SangfA to CypA Potential interacting energies of the three ligands to CypA and its mutants were predicted (Table 2). Positive correlations have been observed in Vina results (after converting results from $\mathrm{Kj} / \mathrm{mol}$ to $\mathrm{Kcal} / \mathrm{mol}$ ). Higher (greater negativity) Lenard-Jones potential (LJ) has been observed in the interaction of SangfA to W121A, HBF-0259 to M61, and SCY-635 to H70F. However, G150F, H70F, and $\mathrm{W} 121 \mathrm{~F}$ had higher (more negativity) LJ potential for SangfA, HBF-0259, and SCY635. 
Table 2. Potential interaction energy of SangfA, HBF-0259, and SCY-635 to the receptors $\begin{array}{lll}\text { SangfA HBF-0259 SCY-635 } & \end{array}$

\begin{tabular}{|c|c|c|c|c|c|c|c|c|c|}
\hline Receptors & $\mathbf{L} \mathbf{J}^{\mathbf{a}}$ & Electro $^{b}$ & Total $^{c}$ & $\mathbf{L J}$ & Electro & Total & LJ & Electro & Total \\
\hline CypA & -338.948 & -50.5292 & -389.477 & -131.168 & -10.0851 & -141.253 & -218.584 & -56.1166 & -274.701 \\
\hline CypA_FM & -361.758 & -33.6781 & -395.436 & -132.135 & -8.36469 & -140.5 & -284.364 & -20.6559 & -305.02 \\
\hline CypA_G150F & -364.418 & -39.5006 & -403.919 & -109.57 & -12.1299 & -121.7 & -259.557 & -24.3092 & -283.866 \\
\hline CypA_K125F & -331.607 & -48.7845 & -380.392 & -119.471 & -14.0771 & -133.548 & -241.206 & -94.3705 & -335.577 \\
\hline CypA_W121A & -300.145 & -43.904 & -344.049 & -119.067 & -14.3043 & -133.371 & -211.879 & -44.2877 & -256.167 \\
\hline CypA_W121F & -342.182 & -44.383 & -386.565 & -112.898 & -2.7668 & -115.665 & -285.341 & -22.0066 & -307.348 \\
\hline CypA_H70F & -338.032 & -45.4765 & -383.509 & -133.906 & -10.7886 & -144.695 & -209.084 & -73.2851 & -282.369 \\
\hline CypA_M61F & -320.313 & -30.6996 & -351.013 & -103.35 & $4.24988^{\mathrm{d}}$ & -99.1001 & -271.852 & -33.2254 & -305.077 \\
\hline CypA_R55F & -335.816 & -60.5728 & -396.389 & -119.215 & $2.65239^{d}$ & -116.563 & -250.626 & -6.80832 & -257.434 \\
\hline \multicolumn{10}{|c|}{$\begin{array}{c}{ }^{\mathrm{a}} \text { Lenard Jones Potential }(\mathrm{Kj} / \mathrm{mol}) \\
{ }^{\mathrm{b}} \text { Electrostatic Potential }(\mathrm{Kj} / \mathrm{mol}) \\
{ }^{\mathrm{c}} \text { Sum of LJ and Electro } \\
{ }^{\mathrm{d}} \text { Positive electro energies }\end{array}$} \\
\hline
\end{tabular}

\section{Hydrogen bonds between ligands and} CypA

No hydrogen bonds (HB) existed between HBF-0259 or SCY-635 and CypA. T. Between SangfA and SCY-635, SangfA had a higher HBs ratio with CypA (75:1), G150 (1.27:1), K125 (4.41:1), W121A (1.32:1), W121F (1.01:1), H70 (8.9:1), M61 (10.14:1), and R55 (1.52:1) (Figure 6). Trajectories of complexes with more hydrogen bonding averages have been used for building complexed pdb files with Visual Molecular Dynamic (VMD) v1.9.3 (26) (Figure 7). HB analysis was performed using the UCSF Chimera 1.10.2 software (15). In this regard, frame 58 with $3 \mathrm{HBs,}$ frame 14 with one $\mathrm{HB}$, and frame 56 corresponding to the frame of CypA_FM with one HB have been selected for SangfA, SCY-635, and HBF-0259, respectively. 


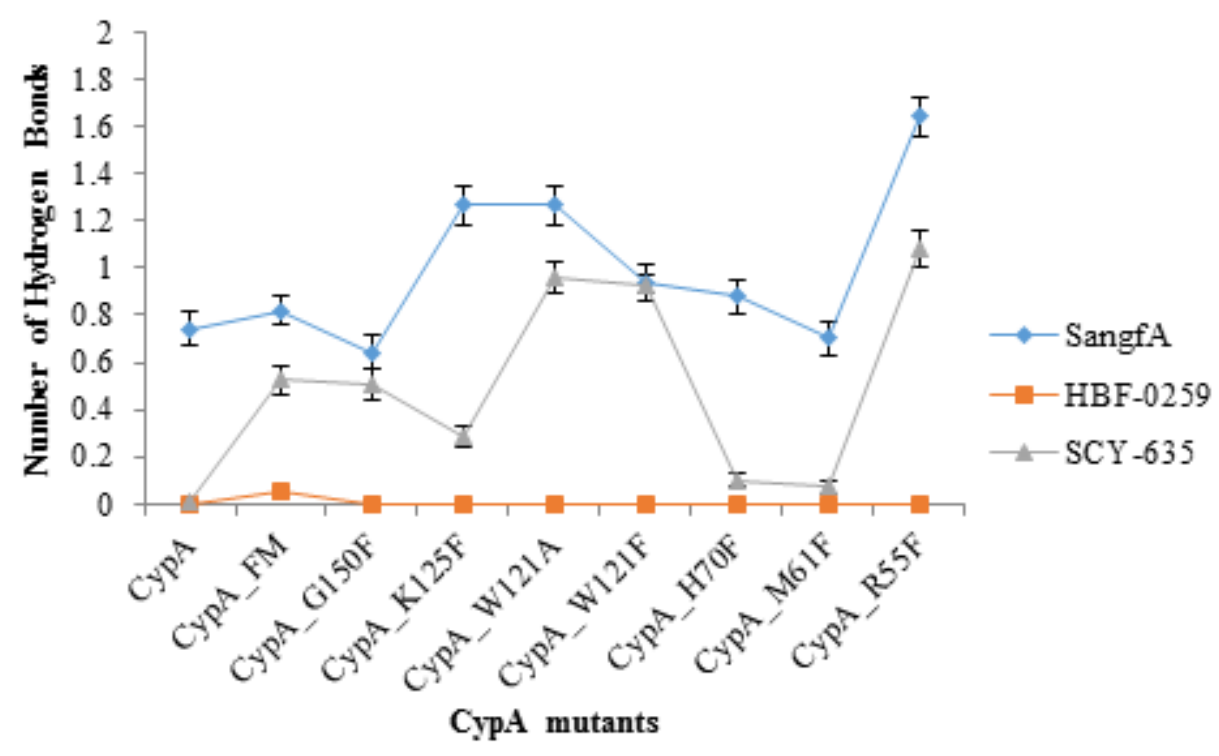

Figure 6. Mean number of hydrogen bonds. Error bars are calculated by 1/10th of the standard deviation 


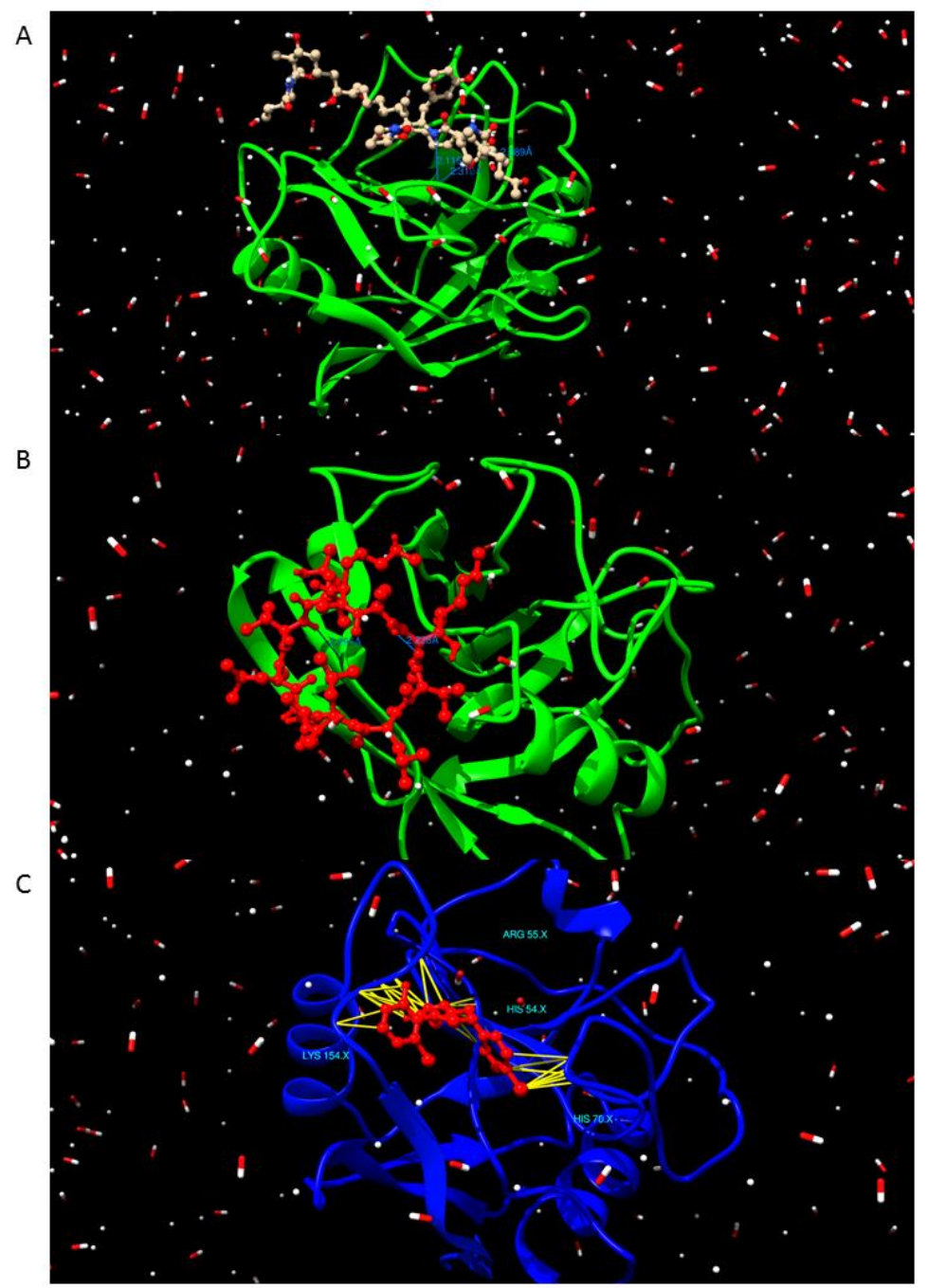

Figure 7. Hydrogen bindings. A) Shows three HBs between Gly109 N and Ligand O166 (2.289 $)$, Thr73 O and Ligand $\mathrm{N}(2.116 \AA)$, and Thr73 O and Ligand $\mathrm{O}(2.316 \AA)$. B) Illustrates HBF-0259 binding site within CypA. Residues that are involved in this electrostatic interaction are Lys154, H70, R55, and H54. C) A schematic representation of two HB between SCY-635 and CypA. In this model, Ala159 O (295 $)$ is involved in intermolecular interaction.

\section{DISCUSSION}

CypA has an essential role in protein folding, transport and intracellular signaling. The importance of CypA has been studied in several viral infections. There are some known inhibitors, which have the potential to bind and inhibit CypA. However, their affinities, post-binding conformational and flexibility changes of CypA as well as their relative toxicities and interaction with the CypA mutants, remain unclear. The present study aimed to answer these questions.
McGowan et al. used molecular dynamics simulations to study human CypA to understand the role of enzyme motions in the catalytic mechanism and recognition (27). They showed that A Trp121Phe mutation, which abolishes this hydrogen bonding interaction, causes CypA to bind cyclosporine with a much lower affinity (27).

The RMSD is a good measure for investigating structural similarities and 
conformational changes in proteins of the same size (28). MD analysis of CypA showed a relative increase in RMSD in W121A and R55F substitutions compared to CypA itself over time. Two-way ANOVA was performed for investigating similarity changes in CypA conformation. In comparison to CypA, no significant conformational changes were observed in W121A/F mutants. Other substitutions had significant RMSD changes. W121A/F substitutions do not induce these conformational changes. Dietrich et al. reported that W121F substitution impairs the interaction of the viral protein to CypA (29). The residue also determines the binding of Csp to CypA ( $\underline{30})$. However, residues like R55 are known to be involved in the isomerase activity of CypA (1ㅡ). Therefore, W121 substitution would be only involved in inhibitors binding to CypA.

The result of Autodock Vina showed SangfA and HBF-0259 had more affinities to the receptor. Accordingly, SangfA with $389.477 \mathrm{Kcal} / \mathrm{mol}$ total energy had the highest intermolecular interaction energy with CypA. Hydrogen bonding was observed in the complex of CypA and SangfA, which was not detected in HBF0259 and SCY-635. Oxygen and two nitrogen atoms of G109 and T73 were acting as $\mathrm{H}$-donors in the complex of CypA and SangfA. These results show that SangfA is more potent than other known inhibitors to interact with CypA, and its interaction is supported by hydrogen bindings, electrostatic forces and hydrophobic effects.

SangfA, HBF-0259 and SCY-635 inhibitors were shown to increase RMSD and decrease Rg. The results also demonstrated that SangfA RMSD was significantly more stable than HBF-0259 and SCY-635. Evaluation of these three inhibitors indicated that CypA complexed with SangfA has more stable RMSD $(\mathrm{p}<0.05)$. This will support no significant conformational changes following SangfA and CypA interaction.

It is well-established that $\mathrm{Rg}$ can evaluate any modification in protein shape (32). This parameter provides information about protein flexibility. Accordingly, Rg values of different CypA mutants were compared to the protein itself. No flexibility changes were observed in $\mathrm{W} 121 \mathrm{~A} / \mathrm{F}$ and $\mathrm{R} 55 \mathrm{~F}$ mutants. Notably, the Rg value in W121A was relatively decreased. Other substitutions had significant changes in $\mathrm{Rg}$ values. This is an indication of the limitation of main chain movement due to substitution. Besides, the flexibility of interacting molecules varied significantly, especially the chains involved in the interactions.

It has been shown that some unique CypA inhibitors could bind to the allosteric site of CypA, which is comprised of Cys52, His70, His54, Lys151, Thr152, and Lys155 residues (11). However, Arginine 55 residue is located in the active site of CypA, where it is involved in the protein PPIase activity (1). Therefore, it acts as an anchor for the substrate in the binding site by preferentially stabilizing the trans and cis isomers' transition state. Consequently, mutation of R55 would conduct at reduction of the catalytic efficiency (33). We assessed total interaction energy (Etot) by summing LJ and electrostatic potentials values. Our electrostatics potentials assessment showed that SangfA had more Etot to CypA ($389.44 \mathrm{Kj} / \mathrm{mol})$. SangfA had higher Etot for CypA_K150F (-403.919) and lower Etot for W121A (-344.049). Since tryptophan 121 residue is essential for the ligand-binding site of CypA (2), this might be a consequence of entropy changes during substitutions at the binding site of CypA. In this regard, Ladani et al. have shown that entropy changes lead to reduced free binding energy at CypA active site (34).

\section{CONCLUSION}

Our results suggest that SangfA could be a more effective inhibitor for CypA. Its interaction with the CypA at the catalytic site does not induce significant vital changes and stability. It was also found that W121 is involved in ligand binding. This was supported by no significant impact of 
W121A/F on CypA conformation and reduced interaction energy of SangfA to the CypA mutant.

\section{ACKNOWLEDGMENTS}

This study was carried out as part of a research approved by the Student Research Committee, School of Medicine, Golestan University of Medical Sciences, Gorgan, Iran (grant number: 970913172).

\section{DECLARATIONS \\ Funding}

This study was supported by the Golestan University of Medical Sciences (grant number: 970913172).

\section{Ethics approvals and consent to participate}

Not applicable

\section{Conflict of interest}

The authors declare that there is no conflict of interest.

\section{REFERENCES}

1. Piotukh K, Gu W, Kofler M, Labudde D, Helms V, Freund C. Cyclophilin A binds to linear peptide motifs containing a consensus that is present in many human proteins. $J$ Biol Chem. 2005;280(25):23668-74. [View $\underline{\text { at Publisher] [DOI] [PubMed] [Google }}$ $\underline{\text { Scholar] }}$

2. Zydowsky LD, Etzkorn FA, Chang HY, Ferguson SB, Stolz LA, Ho SI, et al. Active site mutants of human cyclophilin A separate peptidyl - prolyl isomerase activity from cyclosporin A binding and calcineurin inhibition. Protein Sci. 1992;1(9):1092-9. [View at Publisher] [DOI] [PubMed] [Google Scholar]

3. Nigro P, Pompilio G, Capogrossi MC. Cyclophilin A: A key player for human disease. Cell Death Dis. 2013;4(10). [View $\underline{\text { at Publisher] [DOI] [PubMed] [Google }}$ Scholar]
4. Hopkins S, Dimassimo B, Rusnak P, Heuman D, Lalezari J, Sluder A, et al. The cyclophilin inhibitor SCY-635 suppresses viral replication and induces endogenous interferons in patients with chronic $\mathrm{HCV}$ genotype 1 infection. $J$ Hepatol. 2012;57(1):47-54. [View at Publisher] [DOI] [PubMed] [Google Scholar]

5. Hopkins S, Bobardt M, Chatterji U, Garcia-Rivera JA, Lim P, Gallay PA. The cyclophilin inhibitor SCY-635 disrupts hepatitis $\mathrm{C}$ virus NS5A-cyclophilin a complexes. Antimicrob Agents Chemother. 2012;56(7):3888-97. [View at Publisher] [DOI] [PubMed] [Google Scholar]

6. Hopkins S, Scorneaux B, Huang Z, Murray MG, Wring S, Smitley $C$, et al. SCY-635, a novel nonimmunosuppressive analog of cyclosporine that exhibits potent inhibition of Hepatitis $\mathrm{C}$ virus RNA replication in vitro. Antimicrob Agents Chemother. 2010;54(2):660-72. [View at Publisher] [DOI] [PubMed] [ [ Scholar]

7. Ahmed-Belkacem A, Colliandre L, Ahnou N, Nevers Q, Gelin M, Bessin Y, et al. Fragment-based discovery of a new family of non-peptidic small-molecule cyclophilin inhibitors with potent antiviral activities. Nat Commun. 2016;7. [View at Publisher] [DOI] [PubMed] [Google $\underline{\text { Scholar] }}$

8. Naoumov N V. Cyclophilin inhibition as potential therapy for liver diseases. $\mathrm{J}$ Hepatol. 2014;61(5):1166-74. [View at Publisher] [DOI] [PubMed] [Google Scholar]

9. Mohebbi A, Mohammadi S, Memarian A. Prediction of HBF-0259 interactions with hepatitis B Virus receptors and surface antigen secretory factors. VirusDisease. 2016;27(3):234-41. [View at Publisher] [DOI] [PubMed] [Google Scholar] 
10. Śledź P, Caflisch A. Protein structurebased drug design: from docking to molecular dynamics. Curr Opin Struct Biol. 2018;48:93-102. [View at Publisher] [DOI] [PubMed] [Google Scholar]

11. Lv M, Shi T, Mao X, Li X, Chen Y, Zhu J, et al. 1-(2,6-Dibenzyloxybenzoyl)-3(9H-fluoren-9-yl)-urea: A novel cyclophilin A allosteric activator. Biochem Biophys Res Commun. 2012;425(4):938-43. [View at Publisher] [DOI] [PubMed] [Google $\underline{\text { Scholar] }}$

12. Ivery MTG. A proposed molecular model for the interaction of calcineurin with the cyclosporin A-Cyclophilin a complex. Bioorganic Med Chem. 1999;7(7):1389-402. [View at Publisher] [DOI] [PubMed] [Google Scholar]

13. Berman HM, Battistuz T, Bhat TN, Bluhm WF, Bourne PE, Burkhardt K, et al. The protein data bank. Acta Crystallogr Sect D Biol Crystallogr. 2002;58(6 I):899-907. [View at Publisher] [DOI] [PubMed] [Google Scholar]

14. Kallen J, Mikol V, Taylor P, D.walkinshaw M. X-ray structures and analysis of 11 cyclosporin derivatives complexed with cyclophilin A. J Mol Biol. 1998;283(2):435-49. [View at Publisher] [DOI] [PubMed] [Google Scholar]

15. Pettersen EF, Goddard TD, Huang CC, Couch GS, Greenblatt DM, Meng EC, et al. UCSF Chimera - A visualization system for exploratory research and analysis. J Comput Chem. 2004;25(13):1605-12. [View at Publisher] [DOI] [PubMed] [Google Scholar]

16. Bolton EE, Wang Y, Thiessen PA, Bryant SH. Chapter 12 PubChem: Integrated Platform of Small Molecules and Biological Activities. Annu Rep Comput Chem. 2008;4:217-41. [View at Publisher] [DOI] [Google Scholar]
17. Guex N, Peitsch MC. SWISS-MODEL and the Swiss-PdbViewer: An environment for comparative protein modeling. [View at Publisher] [DOI] [PubMed] [Google Scholar]

18. Chase F, Avenue B. Rotamer libraries in the 21 st century Roland L Dunbrack Jr. Proteins [Internet]. 2002;12(4):431-40. Available from: http://linkinghub.elsevier.com/retrieve/pii/S 0959440X02003445[View at Publisher] [DOI] [Google Scholar]

19. Najmanovich R, Kuttner J, Sobolev V, Edelman M. Side-chain flexibility in proteins upon ligand binding. Proteins Struct Funct Genet. 2000;39(3):261-8. [View at Publisher] [DOI] [PubMed] [Google Scholar]

20. Liu J, Chen CM, Walsh CT. Human and Escherichia coli Cyclophilins: Sensitivity to Inhibition by the Immunosuppressant Cyclosporin A Correlates with a Specific Tryptophan Residue. Biochemistry. 1991;30(9):2306-10. [View at Publisher] [DOI] [PubMed] [Google Scholar]

21. Allouche A. Software News and Updates Gabedit - A Graphical User Interface for Computational Chemistry Softwares. J Comput Chem. 2012;32:17482. [View at Publisher] [DOI] [PubMed] [Google Scholar]

22. Abraham MJ, Murtola T, Schulz R, Páll S, Smith JC, Hess B, et al. Gromacs: High performance molecular simulations through multi-level parallelism from laptops to supercomputers. SoftwareX. 2015;1-2:1925. [View at Publisher] [DOI] [Google Scholar]

23. Oostenbrink C, Villa A, Mark AE, Van Gunsteren WF. A biomolecular force field based on the free enthalpy of hydration and 
solvation: The GROMOS force-field parameter sets 53A5 and 53A6. J Comput Chem. 2004;25(13):1656-76. [View at Publisher] [DOI] [PubMed] [Google Scholar]

24. Schüttelkopf AW, Van Aalten DMF. PRODRG: A tool for high-throughput crystallography of protein-ligand complexes. Acta Crystallogr Sect D Biol Crystallogr. 2004;60(8):1355-63. [View at Publisher] [DOI] [PubMed] [Google $\underline{\text { Scholar] }}$

25. Ihaka R, Gentleman R. R: A Language for Data Analysis and Graphics. J Comput Graph Stat. 1996;5(3):299-314. [View at Publisher] [DOI] [Google Scholar]

26. Humphrey W, Dalke A, Schulten K. VMD: Visual molecular dynamics. J Mol Graph. 1996;14(1):33-8. [View at Publisher] [DOI] [Google Scholar]

27. McGowan LC, Hamelberg D. Conformational plasticity of an enzyme during catalysis: Intricate coupling between cyclophilin a dynamics and substrate turnover. Biophys J. 2013;104(1):216-26. [View at Publisher] [DOI] [PubMed] [Google Scholar]

28. Sargsyan K, Grauffel C, Lim C. How Molecular Size Impacts RMSD Applications in Molecular Dynamics Simulations. J Chem Theory Comput. 2017;13(4):1518-24. [View at Publisher] [DOI] [PubMed] [Google Scholar]

29. Dietrich L, Ehrlich LS, LaGrassa TJ, Ebbets-Reed D, Carter C. Structural Consequences of Cyclophilin A Binding on Maturational Refolding in Human Immunodeficiency Virus Type 1 Capsid
Protein. J Virol. 2001;75(10):4721-33. [View at Publisher] [DOI] [PubMed] [Google Scholar]

30. Lammers M, Neumann H, Chin JW, James LC. Acetylation regulates Cyclophilin A catalysis, immunosuppression and HIV isomerization. Nat Chem Biol. 2010;6(5):331-7. [View at Publisher] [DOI] [PubMed] [Google Scholar]

31. Chatterji U, Bobardt M, Selvarajah S, Yang F, Tang H, Sakamoto N, et al. The isomerase active site of cyclophilin $\mathrm{A}$ is critical for hepatitis C virus replication. J Biol Chem. 2009;284(25):16998-7005. [View at Publisher] [DOI] [PubMed] [Google Scholar]

32. Ciccone DS, Natelson BH. Comorbid illness in women with chronic fatigue syndrome: A test of the single syndrome hypothesis. Psychosom Med. 2003;65(2):268-75. [View at Publisher] [DOI] [PubMed] [Google Scholar]

33. Hamelberg D, Mecammon JA. Mechanistic insight into the role of transition-state stabilization in cyclophilin A. J Am Chem Soc. 2009;131(1):147-52. [View at Publisher] [DOI] [PubMed] [Google Scholar]

34. Ladani ST, Hamelberg D. Entropic and surprisingly small intramolecular polarization effects in the mechanism of cyclophilin A. J Phys Chem B. 2012;116(35):10771-8. [View at Publisher] [DOI] [PubMed] [Google Scholar]

How to Cite: Mohebbi A, Mirarab A, Shaddel R, Shafaei Fallah M, Memarian A. Molecular Dynamic Simulation and Docking of Cyclophilin A Mutants with its Potential Inhibitors. Journal of Clinical and Basic Research 2021; 5 (2):26-41 